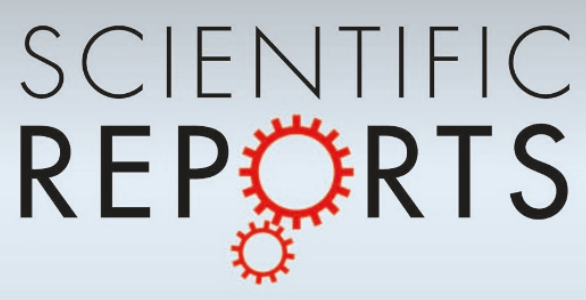

\title{
Compressed carbon nanotubes: A family of new multifunctional carbon allotropes
}

SUBJECT AREAS:

STRUCTURAL MATERIALS

THEORY AND COMPUTATION

CONDENSED-MATTER PHYSICS

SYNTHESIS

Received

1 October 2012

Accepted

7 February 2013

Published

25 February 2013

Correspondence and requests for materials should be addressed to B.X. (bxu@ysu.edu.cn) or Y.J.T. (fhcl@ysu.edu.

cn)

* These authors contributed equally to this work.

\$ Current address: High Pressure

Collaborative Access

Team, Geophysical Laboratory, Carnegie Institution of

Washington, Argonne, Illinois 60439, USA.
Meng Hu' ${ }^{*}$, Zhisheng Zhao ${ }^{1 *}$, Fei Tian ${ }^{2 *}$, Artem R. Oganov', Qianqian Wang', Mei Xiong', Changzeng Fan', Bin Wen', Julong He', Dongli Yu', Hui-Tian Wang'², Bo Xu' \& Yongiun Tian'

'State Key Laboratory of Metastable Materials Science and Technology, Yanshan University, Qinhuangdao 066004, China, ${ }^{2}$ School of Physics and Key Laboratory of Weak-Light Nonlinear Photonics, Ministry of Education, Nankai University, Tianjin 300071, China, ${ }^{3}$ Department of Geosciences, and Department of Physics and Astronomy, Stony Brook University, Stony Brook $11794-2100$, NY, USA.

The exploration of novel functional carbon polymorphs is an enduring topic of scientific investigations. In this paper, we present simulations demonstrating metastable carbon phases as the result of pressure induced carbon nanotube polymerization. The configuration, bonding, electronic, and mechanical characteristics of carbon polymers strongly depend on the imposed hydrostatic/non-hydrostatic pressure, as well as on the geometry of the raw carbon nanotubes including diameter, chirality, stacking manner, and wall number. Especially, transition processes under hydrostatic/non-hydrostatic pressure are investigated, revealing unexpectedly low transition barriers and demonstrating $s p^{2} \rightarrow s p^{3}$ bonding changes as well as peculiar oscillations of electronic property (e.g., semiconducting $\rightarrow$ metallic $\rightarrow$ semiconducting transitions). These polymerized nanotubes show versatile and superior physical properties, such as superhardness, high tensile strength and ductility, and tunable electronic properties (semiconducting or metallic).

arbon has the ability to adopt $s p-, s p^{2}$, and $s p^{3}$-hybridized states, thus forming a wide range of allotropes including graphite, diamond, fullerenes, carbon nanotubes (CNTs), and carbynes. The creation of new multifunctional carbon allotropes has been the focus of numerous theoretical and experimental explorations because of their fundamental scientific and technological importance.

The periodic bonding motif in carbon structures can be dramatically altered under pressure, such as the wellknown $s p^{2} \rightarrow s p^{3}$ bonding change. By compressing different raw carbons such as graphite, fullerenes, CNTs, and vitreous carbon, diverse phases are produced including diamond, transparent superhard post-graphite phases ${ }^{1-4}$, one-, two-, and three-dimensional (3D) fullerene polymers ${ }^{5}$, fully $s p^{3}$-bonded superhard amorphous carbon ${ }^{6}$, and some elusive allotropes ${ }^{7-9}$. However, completely clarifying the structures of these exciting carbon phases through current experimental technologies is usually unrealistic due to their limited quantity, as well as mixture of other phases. State-of-the-art theoretical tools for structure prediction are necessary and have yielded great success, such as simulated annealing ${ }^{10,11}$, genetic algorithm ${ }^{12,13}$, basin hopping ${ }^{14,15}$, metadynamics ${ }^{16,17}$, evolutionary metadynamics $^{18,19}$, random sampling ${ }^{20,21}$, and particle-swarm optimization ${ }^{22,23}$. Most of these algorithms focus on the search of thermodynamically favorable structures with little consideration on the kinetics process, which is also important for metastable materials. The recently developed variable-cell nudged elastic band method ${ }^{18}$ and transition path sampling method ${ }^{24}$ enable further investigations into these kinetic processes.

By considering both thermodynamics and kinetics aspects, we proposed a novel carbon allotrope, namely Cco$\mathrm{C}_{8}{ }^{25}$, which can be viewed as a $3 \mathrm{D}(2,2)$ CNT polymer and account for the previously undetermined superhard carbon phase recovered from cold compression of CNT bundles ${ }^{26}$. Further dynamical simulations demonstrated the easy formation of Cco- $\mathrm{C}_{8}$ by compressing armchair CNT bundles. Using transition path sampling method, we found that for kinetic reasons (minimal transition barrier), room-temperature compression of graphite yields $\mathrm{M}$ carbon, which was first theoretically proposed ${ }^{13,27}$ and very recently unambiguously identified experimentally ${ }^{28}$. These results inspired us to search and identify more new carbon allotropes under pressure with similar considerations. Numerous experimental studies on the high-pressure behavior of CNTs have been performed, most of which focused on the structural, electronic, and mechanical resonances of CNTs under low deformation ratio $^{29,30}$. In this region, the tubes only undergo reversible polygonization, ovalization, or racetrack-shape deformations. Possible pressure-induced polymerizations of CNTs are rarely investigated except for limited cases ${ }^{31-35}$. Multi-walled CNTs (MWCNTs) become partially transparent under a non-hydrostatic pressure of $11 \mathrm{GPa}$ and further transform into a new hard phase with complete $s p^{3}$ bonding under $16 \mathrm{GPa}^{35}$. Under higher non-hydro- 

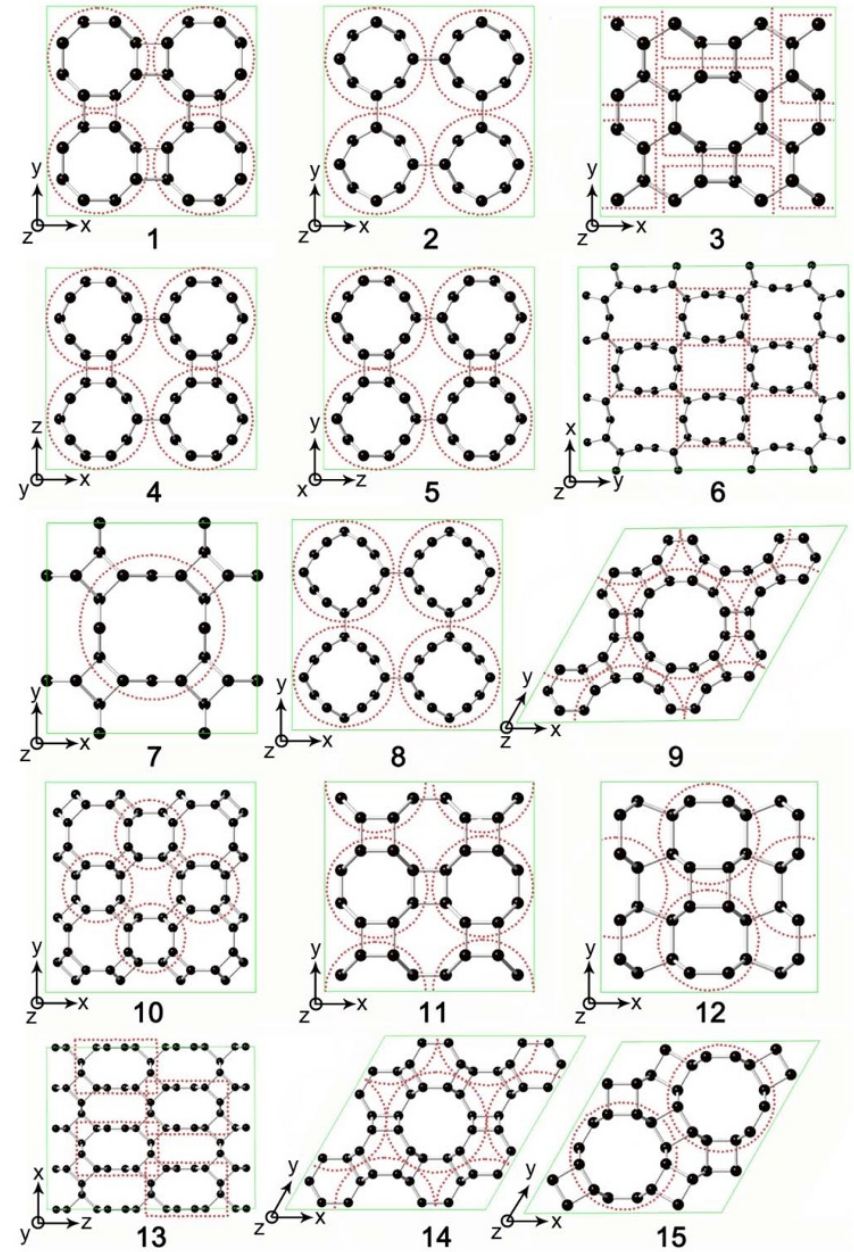

Figure $1 \mid$ Top view of 3D SWCNTs polymers along the axial directions. 1-3: 3D (4,0)-I, -II, and -III; 4-6: 3D (5,0)-I, -II, and -III; 7-9: 3D $(6,0)-I,-I I$, and -III; 10-12: 3D (2,2)-I, -II, and -III; 13-15: 3D (3,3)-I, -II, and -III. The corresponding $x, y$, and $z$ axes of crystal structures are marked. static pressures of 24 and $35 \mathrm{GPa}$, two novel superhard carbon phases with respective hardness of $62-150 \mathrm{GPa}$ and $58 \mathrm{GPa}$ have been synthesized by compressing single-walled CNTs (SWCNTs) with Raman spectral features indicating the polymerization of $\mathrm{CNTs}^{32,34}$. Other elusive allotropes have been obtained upon simultaneous application of temperature and pressure ${ }^{31,33}$. Although these experimental results are motivating, most of the crystal structures of these carbon phases derived from CNTs are unsolved, and the physical picture underneath the pressure-induced phase transitions of nanotubes remains unclear.

In this paper, a systematic and comprehensive study about pressure-induced CNT polymerization is presented via $a b$ initio calculations. Significant changes in structural, electronic, and mechanical properties occur along with CNTs polymerization under pressure, yielding a variety of novel carbon allotropes strongly depending on the applied pressure and the nature of the CNTs precursor. The surprisingly low energy barrier for these transitions indicates a feasible pathway to produce new carbon allotropes with engineered properties.

\section{Results}

Polymerization under pressure. Under hydrostatic pressure, small zigzag $(n, 0)$ and armchair $(n, n)$ SWCNTs can easily bond covalently to each other by directly shortening intertube distance from $3.4 \AA$ to approximately $1.55 \AA$ (Fig. 1). The compressed nanotubes manifest diverse structures related to the CNT species and initial stacking configurations. For example, 3D (4,0)-I and 3D (4,0)-III carbons can be selectively formed due to AAA and ABA stacking of $(4,0)$ SWCNTs, respectively (Fig. S2 in Supplementary Information), whereas the difference between 3D $(4,0)$-I and 3D $(4,0)$-II carbons is due to the different orientations of CNTs inside similar tetragonal unit cells. As a consequence, a great number of metastable carbon structures can be produced by compressing small SWCNTs in our simulations. Some previously proposed structures, such as $3 \mathrm{D}(2,2)-$ II $\left(\mathrm{Bct}_{-} \mathrm{C}_{4}{ }^{36}\right), 3 \mathrm{D}(2,2)$-III $\left(\mathrm{CcO}-\mathrm{C}_{8}{ }^{25}\right), 3 \mathrm{D}(5,0)-\mathrm{III}^{37}$, and $3 \mathrm{D}(3,3)-\mathrm{I}^{38}$, have been identified along with more new structures. The transition pressures for small $(n, 0)$ SWCNTs are usually less than $20 \mathrm{GPa}$, whereas those for small $(n, n)$ SWCNTs usually exceed $20 \mathrm{GPa}$. This difference can be attributed to the local flattened section in $(n, n)$ SWCNTs, where the $\pi$ bond repulsion prevents further compression (see detailed discussions below). Once this repulsion is overcome by higher pressure, the common $[2+2]$ cycloaddition
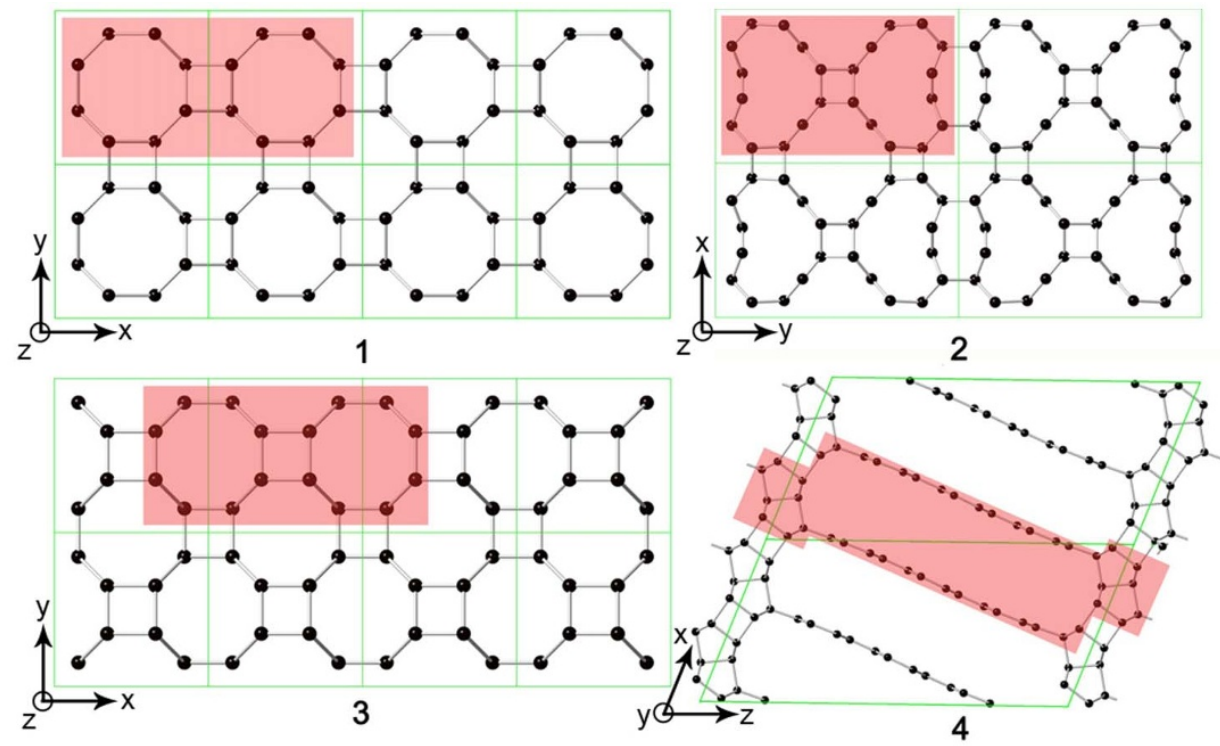

Figure $2 \mid$ Top view of 3D SWCNTs polymers along the axial directions. 1-4:3D (4,0)-I, (6,0)-IV, (2,2)-II, and (8,8), recovered from (8,0), (12,0), (4,4), and $(10,10)$ SWCNTs (red regions), respectively. The corresponding $x, y$, and $z$ axes of crystal structures are marked. 


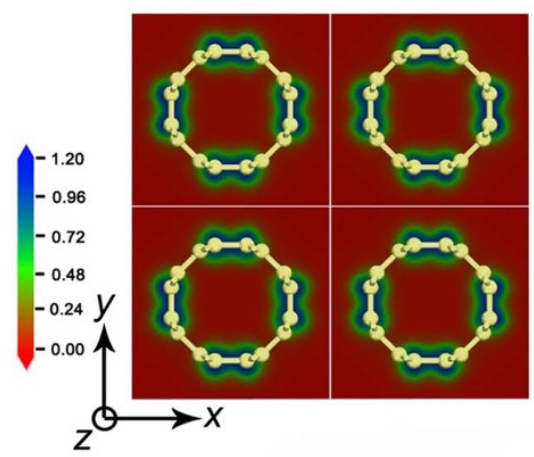

$(4,4) \rightarrow 3 D(4,4)$
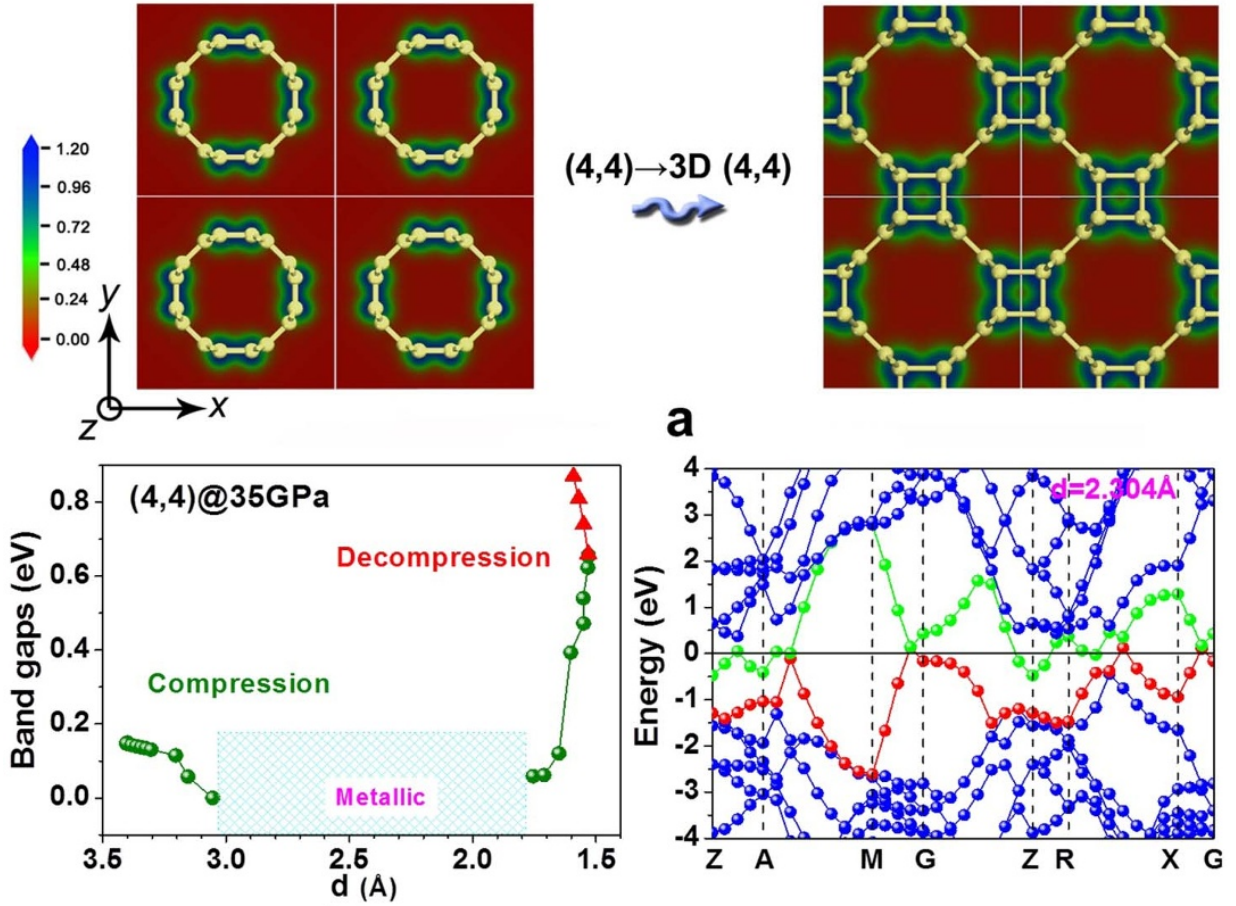

b

C

Figure $3 \mid$ (a): structural evolution from $(4,4)$ SWCNT bundles to 3D $(4,4)$ carbon under hydrostatic pressure of 35 GPa; (b): band gap evolution as a function of intertube distance $d(\AA)$, where olive spheres correspond to the compression process (@35 GPa) and red triangles correspond to the decompression process (@ $0 \mathrm{GPa}$ ); (c): electron band structure at $\mathrm{d}=2.304 \AA$.
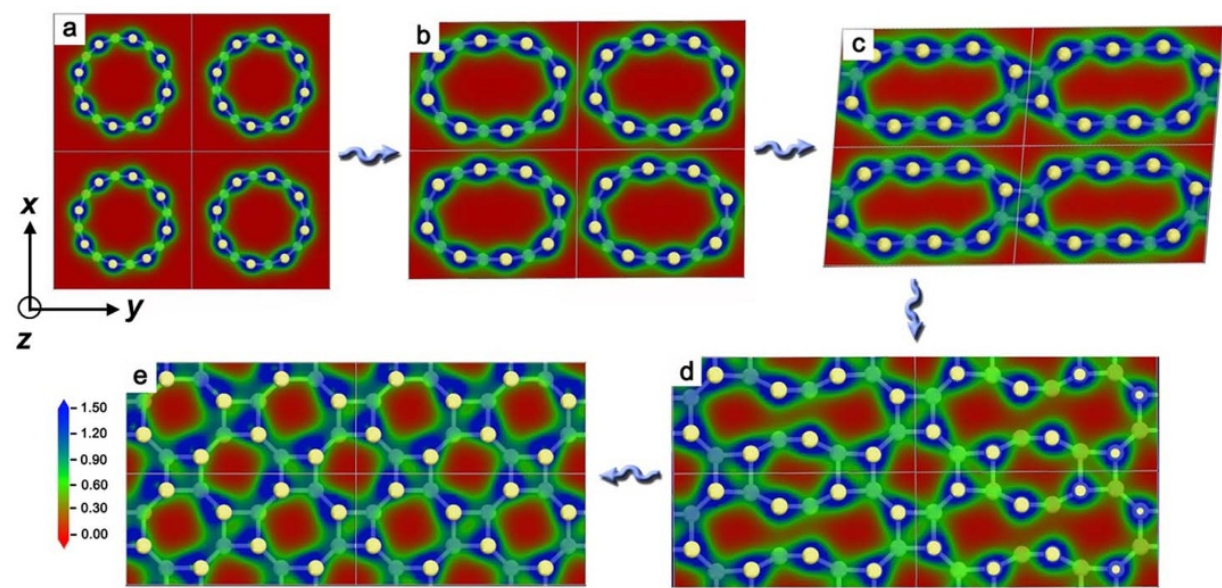

$(8,0) \rightarrow 3 D(4,0)-I$

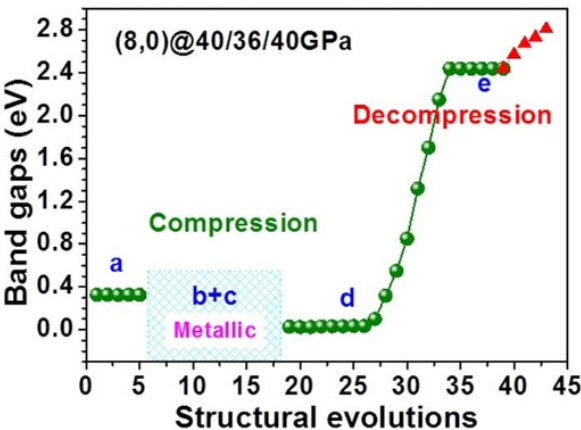

f

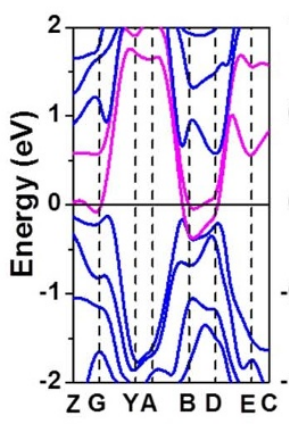

Structure 12

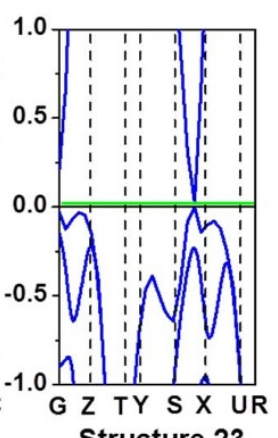

Structure 23

Figure $4 \mid$ (a-e): structural evolution from $(8,0)$ SWCNT bundles to 3D $(4,0)$-I carbon under non-hydrostatic pressure of 40 GPa along $x$ axis and $z$ axis, and $36 \mathrm{GPa}$ along $y$ axis; (f): band gaps as a function of structural evolution in the compression process (olive spheres) and the decompression process (red triangles); (g): band structure of evolution 12 (metallic) and evolution 23 (semiconducting) in panel (f), where the magenta curves correspond to the bands crossing the Fermi level, and the green shadow emphasizes the band gap. 


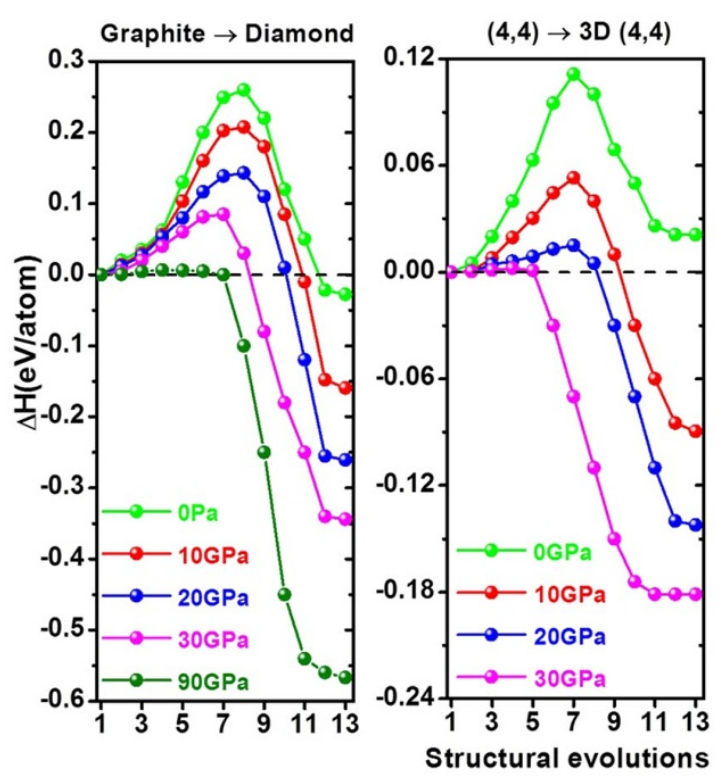

$(8,0) \rightarrow 3 D(4,0)-1$

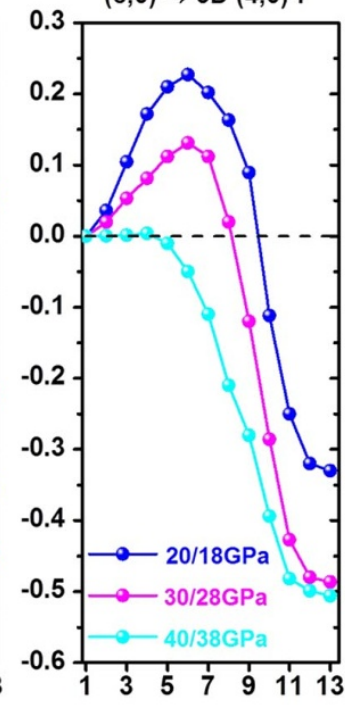

Figure 5 Energy barrier curves from 3R graphite to cubic diamond, aligned $(4,4)$ SWCNTs to 3D $(4,4)$ carbon, and aligned $(8,0)$ SWCNTs to 3D $(4,0)$-I carbon at hydrostatic/non-hydrostatic pressures. Non-hydrostatic A/B GPa indicates the applied pressure is A GPa along $x$ axis and $z$ axis, and B GPa along $y$ axis.

happens at intertube junctions, and four-membered rings appear in $3 \mathrm{D}(n, n)$ SWCNT polymer structures.

Non-hydrostatic pressure shows a much more significant effect on aligned large SWCNTs than on small SWCNTs. Under pressure, large SWCNTs have more variants and can collapse into denser polymers consisting of smaller SWCNTs with higher incompressibility. For example, $(4,4),(8,0)$, and $(12,0)$ SWCNTs are compressed to form $3 \mathrm{D}(2,2)$ (Bct- $\mathrm{C}_{4}{ }^{36}$ and $\left.\mathrm{Cco}_{-} \mathrm{C}_{8}{ }^{25}\right), 3 \mathrm{D}(4,0)$-I, and $3 \mathrm{D}(6,0)-\mathrm{IV}$ carbons, respectively (See Fig. 2). Further investigations on compression of larger nanotubes showed that more complicated structures can be obtained from complicated deformations of tube cross sections. For instance, aligned $(10,10)$ SWCNTs under non-hydrostatic pressure can first transform into $3 \mathrm{D}(8,8) \mathrm{CNT}$ polymer (Fig. 2), and then form a bond in the local graphite-like section of the structure under increasing pressure. The extremely complex phase behavior in compressed CNTs is fundamentally determined by the structural diversity of CNTs. It should be noted that, except for the successful assignment of $\mathrm{Cco}-\mathrm{C}_{8}$ to a cold compressed $\mathrm{CNTs}^{25,26}$, direct comparisons between our simulated CNT polymers and other synthesized phases are hard to carry out at present. Most of experimental studies did not provide enough structural information ${ }^{31-35}$. In addition, the starting materials in these experiments are usually a mixture of different CNTs, making such a comparison even harder. A better control of starting SWCNT bundles, such as improved alignment and chirality/diameter purification, may make such comparisons possible in the future.

Structural evolution. Although many experiments have suggested the structural transition of nanotubes under pressure, such as deformation, collapse, and polymerization ${ }^{29,30}$, the kinetic mechanism of transition has not been revealed up to our knowledge. To elaborate the kinetics process of phase transition, we investigated the structure evolutions of aligned $(4,4)$ and $(8,0)$ SWCNTs under hydrostatic and non-hydrostatic pressure, respectively (Figs. 3 and 4). For aligned $(4,4)$ SWCNTs, adjacent nanotubes interact by weak van der Waals interactions at ambient pressure. A band gap is open at Fermi level, which is distinct from metallic isolated $(n, n)$ SWCNTs $^{39}$ but consistent with the theoretical prediction $^{40}$ and experimental observation ${ }^{39}$ on $(n, n)$ SWCNT bundles. After applying pressure, the intertube distance in aligned
$(4,4)$ SWCNTs is shortened, leading to increased intertube $\pi-\pi$ coupling. The $(4,4)$ SWCNTs polymerize to form 3D $(4,4)^{38}$ carbon, accompanied by $\pi \rightarrow \sigma$ bonding conversion in intertube junctions under higher pressure. Such simple structural changes cause unexpectedly semiconducting $\rightarrow$ metallic $\rightarrow$ semiconducting electronic changes, as shown in Fig. $3 \mathrm{~b}$. When intertube distance decreases from standard $3.4 \AA$ to $3.054 \AA$, enhanced intertube $\pi-\pi$ coupling induces a downward shift of the green conduction band (Fig. 3c) near the Fermi level and closes the band gap from $0.16 \mathrm{eV}$ to $0 \mathrm{eV}$ (Fig. S3). Further shortening of intertube distance continuously moves the green conduction lower and the red valence band higher across the Fermi level, resulting in a conductive behavior of the compressed $(4,4)$ tubes (Figs. 3c and S3). In this "metallic" region, the electronic total density of states (DOS) at the Fermi level is determined by the green and red bands across the Fermi level and demonstrates a non-monotonic variation. This observation is reminiscent of an earlier work about the hypothetical metallic $\mathrm{ThSi}_{2}$-structured carbon, where the metallicity was attributed to short $\pi-\pi$ contacts $^{41}$. During the final stage of compression, as soon as an intertube distance of $1.804 \AA$ is reached, a sudden $\pi \rightarrow \sigma$ intertube bonding transition occurs companied with valence electron localization. A band gap would re-open at the Fermi level and gradually increase to $0.66 \mathrm{eV}$ as the length of the $\sigma$-bond is shortened to $1.531 \AA$. Moreover, this $3 \mathrm{D}(4,4)$ carbon is quenchable with the band gap increasing from $0.66 \mathrm{eV}$ to $0.87 \mathrm{eV}$ after decompressing from $35 \mathrm{GPa}$ to ambient pressure.

The structural change of aligned $(8,0)$ SWCNTs under non-hydrostatic pressure is more complicated than that of $(4,4)$ SWCNTs under hydrostatic pressure. Moreover, the corresponding electronic changes are more intriguing. Specifically, the aligned $(8,0)$ SWCNTs undergo four major deformations (Fig. 4) under nonhydrostatic pressure. In the first stage, the initially circular crosssection of the nanotubes becomes oval-shaped (Fig. 4b), a phenomenon which has been previously extensively studied ${ }^{42,43}$. This deformation is reversible and induces a semiconductor $\rightarrow$ metal transition in compressed $(8,0)$ nanotubes, consistent with previous investigation of isolated $(7,0)$ nanotubes ${ }^{44}$. In the second stage, 1D $(8,0)$ SWCNTs start to polymerize into $2 \mathrm{D}(8,0)$ nanotube polymers (hereafter named 2D $(8,0)$ carbon) (Fig. 4c), similar to previous simulations of $2 \mathrm{D}(5,0),(7,0)$, and $(9,0)$ carbon $^{37}$. The $2 \mathrm{D}(8,0)$ 

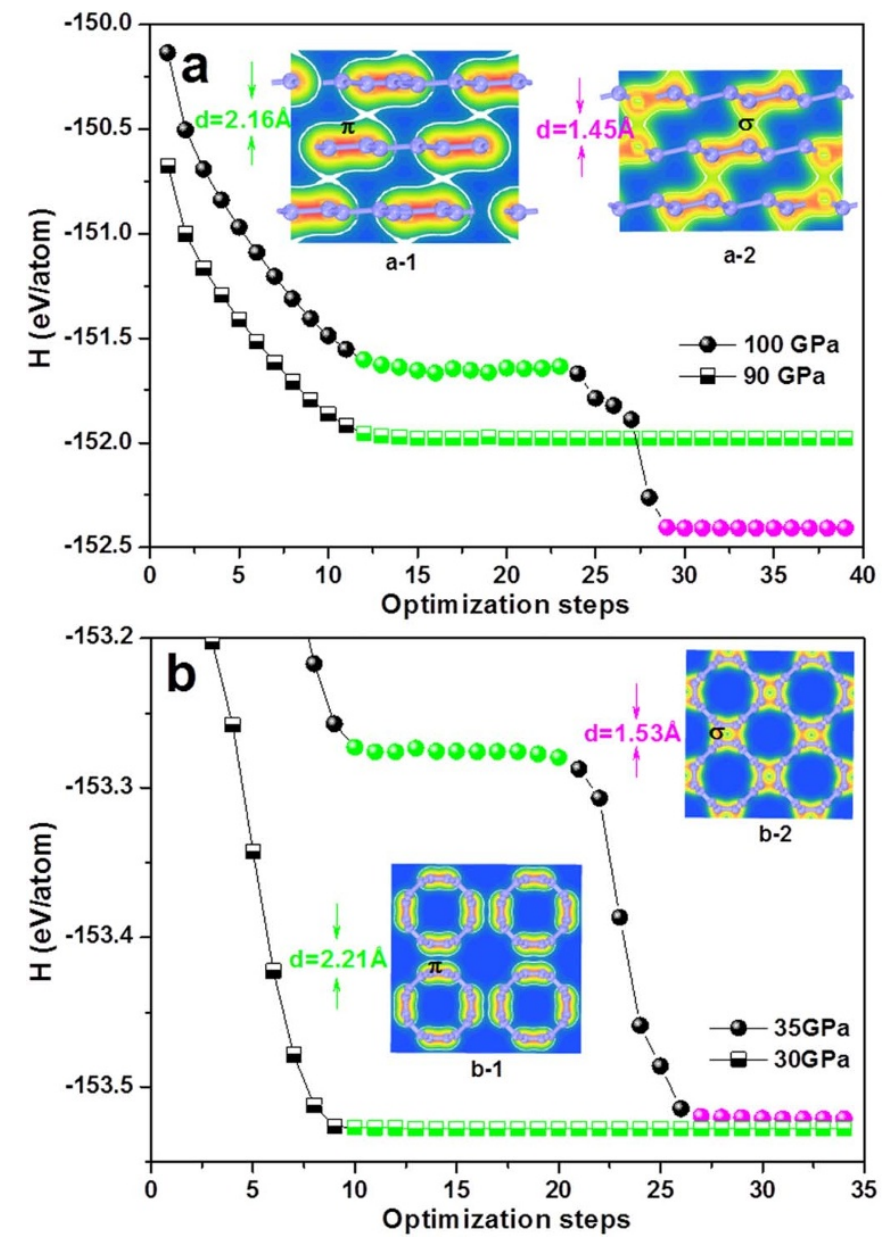

Figure 6 | Enthalpy $(\mathrm{H})$ changes as a function of optimization steps under different pressures. (a) and (b): phase transitions of $3 \mathrm{R}$ graphite $\rightarrow 3 \mathrm{C}$ diamond and $(4,4) \rightarrow 3 \mathrm{D}(4,4)$ carbon, respectively. Insets (a-1) and (b-1) show the electron density profiles corresponding to the green marks in the curves, while insets (a-2) and (b-2) show the electron density profiles corresponding to the magenta spheres in the curves. A phase transition only occurs after a critical pressure being applied (100 GPa for graphite and 35 GPa for $(4,4)$ CNT). carbon remains metallic because the stronger $\pi-\pi$ coupling contributes more delocalized electrons. In the third stage, $2 \mathrm{D}(8,0)$ carbon transforms into $3 \mathrm{D}(8,0)$ carbon through further intertube bond formation (Fig. 4d). More electrons are localized in this stage, leading to a semiconductor with a very narrow band gap $(c a .0 .02 \mathrm{eV})$. In the final stage, $3 \mathrm{D}(8,0)$ carbon is further squashed to form fully $s p^{3}$ bonded denser 3D (4,0)-I carbon (Fig. 4e). As a result, the band gap rapidly increases up to $2.4 \mathrm{eV}$. This $3 \mathrm{D}(4,0)$-I carbon is quenchable and the band gap increases to $2.8 \mathrm{eV}$ after decompressing the pressure. This band gap oscillation is not unique, and different transition paths would lead to different electronic property changes.

Low transition energy barrier. Although the enthalpy of CNT polymer is lower than that of its counterpart CNT, a central issue to rationalize such phase transition is pertinent to the transition barrier (Fig. 5). The result of $3 \mathrm{R}$ graphite $\rightarrow 3 \mathrm{C}$ diamond is also presented in Fig. 5 for comparison. This transition can easily be realized by directly shortening the graphite interlayer distance and buckling the neighboring sheets. The calculated energy barriers decrease with increasing pressure. The values of 0.26 and $0.14 \mathrm{eV} /$ atom at 0 and $20 \mathrm{GPa}$ respectively are in good agreement with previously calculated $0.33^{45}$ and $0.15^{46} \mathrm{eV} /$ atom, indicating the rationality of our method (See Methods section for details). The pressure-induced phase transition of $(4,4)$ SWCNT $\rightarrow 3 \mathrm{D}(4,4)$ is also simple. The calculated transition barrier for this transition is notably lower than that of graphite $\rightarrow$ diamond transition at the same pressure, implying a more feasible transition process. For $(8,0)$ SWCNTs $\rightarrow$ DD $(4,0)$-I transition, the energy barrier at 20/18 GPa pressure is higher than that of graphite $\rightarrow$ diamond at $20 \mathrm{GPa}$. However, it rapidly decreases with increasing pressure and drops to $0 \mathrm{eV}$ at $40 / 38 \mathrm{GPa}$, whereas $90 \mathrm{GPa}$ is needed to drop the barrier to $0 \mathrm{eV}$ for graphite $\rightarrow$ diamond.

In compressed graphite or $(4,4)$ SWCNT, the highest energy-barrier points correspond to a specific structure with interlayer or intertube spacing of $c a$. $2.2 \AA$. For instance, the relaxed equilibrium structure at $90 \mathrm{GPa}$ is still graphite-like with an interlayer distance of about $2.2 \AA$ because this much shortened distance induces strong $\pi$ bond repulsion to resist external pressure (Fig. 6a). At $0 \mathrm{~K}$, graphite remains to be metastable until a critical pressure of $100 \mathrm{GPa}$ is applied, where $\pi-\pi$ repulsion is conquered and $\pi \rightarrow \sigma$ bonding transition occurs accompanied by the structural change and a large enthalpy drop. By comparison, a critical pressure of as low as 35 $\mathrm{GPa}$ is sufficient to overcome the $\pi-\pi$ repulsion in compressed $(4,4)$ SWCNTs, far less than that of graphite (Fig. 6b). These results clearly indicate the easy formation of new metastable carbons

Table 1 The calculated energy band gap (eV), axial and radial Young's modulus $Y_{\mathrm{a}}$ and $Y_{\mathrm{r}}(\mathrm{TPa})$, Vickers hardness $H_{v}(G P a)$, axial and radial tensile strength $\sigma_{a}$ and $\sigma_{r}(\mathrm{GPa})$, bulk modulus $B(\mathrm{GPa})$, shear modulus $G(\mathrm{GPa}), B / G$ radio, and Poisson's ratio $v$

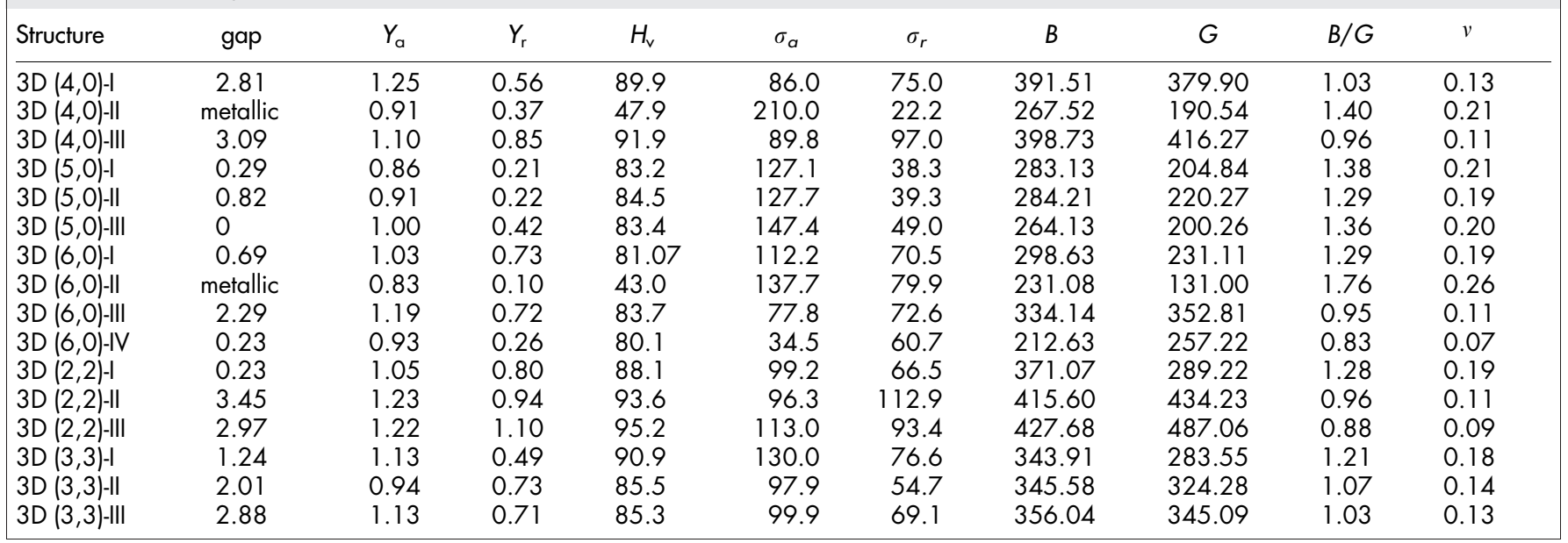


through compressing small-diameter CNTs, compared with the case of graphite compression.

Electronic and mechanical properties. Band structure calculations show that $3 \mathrm{D}(4,0)$-II, $3 \mathrm{D}(6,0)$-II, and $3 \mathrm{D}(8,8)$ carbons are metallic and other carbon polymers are semiconducting with wide direct/ indirect gaps from $0 \mathrm{eV}$ to $3.45 \mathrm{eV}$ (Table 1 and Fig. S4). CNTs process excellent mechanical properties, such as high stiffness and tensile strength, along axial direction. They are easily deformable in the radial direction which limits their application. Polymerization would greatly improve the mechanical properties in the radial direction without much loses along the axial one. The 3D CNT polymers have obviously larger Young's modulus, bulk modulus, and shear modulus compared with their 1D counterpart (Tables 1 and S5). We also estimated the hardness and tensile strength of carbon polymers based on our developed models ${ }^{47-51}$. The results indicate that $3 \mathrm{D}$ polymers composed of small CNTs are all superhard carbons, among which metallic carbons have lower hardness (Tables 1 and S2). Notably, our hardness model may not be suitable for 3D polymers composed of large CNTs with very high pores because the prevailing $s p^{2}$-hybridized bonds are flexible and may not be destroyed in response to external stress. Our tensile strength model can be applied to all carbon polymers. The 3D CNT polymers have high axial tensile strength comparable to that of $\mathrm{CNTs}^{52}$, and have high radial tensile strength due to $s p^{3}$-bond bucklings along the radial directions (Tables 1, S3 and S4). The Poisson's ratio ${ }^{53}$ and $\mathrm{B} / \mathrm{G}^{54}$ values of these carbon allotropes indicate that they are more ductile (less brittle) than diamond and lonsdaleite. An assembly of these excellent physical properties would make 3D CNT polymers ultimate materials for multipurpose applications.

\section{Discussion}

Carbon allotropes are notable due to the diversity of their structures and properties. Syntheses of these novel carbon phases depend on two important aspects of the transition process, namely the enthalpies of the initial and final states and the transition energy barrier. CNTs are a suitable choice of precursors because they possess high enthalpy with respect to graphite. On the other hand, the transition barrier can be well tuned with pressure: a lower energy barrier can be achieved under higher pressure ${ }^{55}$. We thus proposed an effective route to achieve numerous novel carbons with versatile physical properties, namely the pressure induced CNT polymerization. Specifically, the structural, electronic, and mechanical property changes of small SWCNTs, such as $(4,0),(5,0),(6,0),(8,0),(12,0)$, $(2,2),(3,3),(4,4)$, and $(10,10)$ CNTs, under hydrostatic/non-hydrostatic pressure are presented. During the transition, peculiar conductance oscillations in compressed CNTs are revealed in the calculations, an effect that can be used in nanoelectromechanical systems. The estimated transition barriers of small SWCNTs under pressure are less than that of graphite, indicating an easier synthesis of polymeric CNTs. The obtained 3D polymers strongly relate to the raw CNTs with different diameters, chiralities, stacking manners, and wall numbers. These novel 3D CNT polymers show excellent mechanical properties, such as high hardness, strength, and ductility, and a wide range of electronic properties ranging from metallic to semiconducting. Similarly exciting polymorphs may also be accessible from high-pressure studies of CNTs analogs, such as boron nitride, silicon, germanium, and $\mathrm{MoS}_{2}$ nanotubes. As a final remark, we point out the possibility of achieving more exotic carbon allotropes with superior properties from compressing MWCNT bundles as well as mixture of distinct nanotubes, and related simulations are being undertaken.

\section{Methods}

Carbon nanotubes packed in periodic crystal lattices, such as hexagonal, orthorhombic, and tetragonal arrangements, with a standard intertube spacing of 3.4
$\AA$ A were constructed using the Materials Studio package ${ }^{56}$. Structural relaxations and property calculations were performed based on the density functional theory (DFT) as implemented in the CASTEP $\operatorname{code}^{56}$. The Vanderbilt ultrasoft pseudopotential was used and the electron-electron exchange interaction was described by the local density approximation (LDA) exchange-correlation functional of Ceperley and Alder, as parameterized by Perdew and Zunger $(\mathrm{CA}-\mathrm{PZ})^{57,58}$. The plane-wave cutoff energy was $310 \mathrm{eV}$, and a $k$-point spacing $\left(2 \pi \times 0.04 \AA^{-1}\right)$ was used to generate Monkhorst-Pack $k$-points grids for Brillouin zone sampling ${ }^{59}$. Primitive cells were used to calculate the band structures and the elastic constant, Young's modulus, bulk modulus, and shear modulus.

For the transition paths of $3 \mathrm{R}$ graphite $\rightarrow 3 \mathrm{C}$ diamond and $(4,4) \rightarrow 3 \mathrm{D}(4,4)$ under hydrostatic pressure, the intermediate structures are determined by linearly altering the lattice parameters $(a, b, c, \alpha, \beta$, and $\gamma)$ from the initial structures of $3 \mathrm{R}$ graphite and $(4,4)$ to the final structures of $3 \mathrm{C}$ diamond and 3D $(4,4)$, respectively. For example, $a_{i}$ and $a_{f}$ are the lattice parameters of $3 \mathrm{R}$ graphite and $3 \mathrm{C}$ diamond under $30 \mathrm{GPa}$, respectively. After equally divide the difference $a_{f}-a_{i}$ into 50 parts, the lattice parameter of an intermediate structure can be defined as $a_{n}=a_{i}+n\left(a_{f}-a_{i}\right) / 50$, where $n$ running from 1 to 49 represents the $\mathrm{n}^{\text {th }}$ intermediate phase. We then optimize the atomic positions with fixed lattice parameter at the given hydrostatic pressure $(30 \mathrm{GPa})$. Similarly, for the transition path of $(8,0) \rightarrow 3 \mathrm{D}(4,0)$-I under non-hydrostatic pressure, the intermediate structures are determined by linearly altering the atomic fractional coordinates from $(8,0)$ to $3 \mathrm{D}(4,0)$-I. After that, the atomic fractional positions for each intermediate structure are fixed and the lattice is fully optimized. The corresponding enthalpies are then achieved for the optimized structures. The energy barrier can then be deduced from these enthalpies for specific applied pressure. As shown in Figure 5, thirteen typical values are selected to describe the trend of enthalpy and reveal the energy barrier in the transition process under applied pressure.

1. Aust, R. B. \& Drickamer, H. G. Carbon: A new crystalline phase. Science 140, 817-819 (1963)

2. Miller, E. D., Nesting, D. C. \& Badding, J. V. Quenchable transparent phase of carbon. Chem. Mater. 9, 18-22 (1997).

3. Mao, W. L. et al. Bonding changes in compressed superhard graphite. Science 302, 425-427 (2003).

4. Boulfelfel, S. E., Oganov, A. R. \& Leoni, S. Understanding the nature of "superhard graphite" Sci. Rep. 2, 471 (2012)

5. Sundqvist, B. Fullerenes under high pressures. Adv. Phys. 48, 1-134 (1999).

6. Lin, Y. et al. Amorphous diamond: A high-pressure superhard carbon allotrope. Phys. Rev. Lett. 107, 175504 (2011).

7. Hirai, H. \& Kondo, K.-I. Modified phases of diamond formed under shock compression and rapid quenching. Science 253, 772-774 (1991).

8. Tonkov, E. Y. \& Ponyatovsky, E. Phase transformations of elements under high pressure. Vol. 4 (CRC, 2005).

9. El Goresy, A. et al. A new natural, super-hard, transparent polymorph of carbon from the Popigai impact crater, Russia. C. R. Geoscience 335, 889-898 (2003).

10. Kirkpatrick, S., Gelatt, C. D. \& Vecchi, M. P. Optimization by simulated annealing. Science 220, 671-680 (1983).

11. Pannetier, J., Bassas-Alsina, J., Rodriguez-Carvajal, J. \& Caignaert, V. Prediction of crystal structures from crystal chemistry rules by simulated annealing. Nature 346, 343-345 (1990)

12. Glass, C. W., Oganov, A. R. \& Hansen, N. USPEX-evolutionary crystal structure prediction. Comput. Phys. Commun. 175, 713-720 (2006).

13. Oganov, A. R. \& Glass, C. W. Crystal structure prediction using ab initio evolutionary techniques: principles and applications. J. Chem. Phys. 124, 244704-244715 (2006)

14. Wales, D. J. \& Doye, J. P. K. Global optimization by basin-hopping and the lowest energy structures of lennard-jones clusters containing up to 110 atoms. J. Phys. Chem. A 101, 5111-5116 (1997).

15. Nayeem, A., Vila, J. \& Scheraga, H. A. A comparative study of the simulatedannealing and Monte Carlo-with-minimization approaches to the minimum energy structures of polypeptides: [met]-enkephalin. J. Comput. Chem. 12, 594-605 (1991).

16. Martoňák, R., Laio, A. \& Parrinello, M. Predicting crystal structures: the Parrinello-Rahman method revisited. Phys. Rev. Lett. 90, 075503 (2003).

17. Martoňák, R. et al. Simulation of structural phase transitions by metadynamics. Zeitschrift für Kristallographie 220, 489-498 (2005).

18. Zhu, Q., Zeng, Q. \& Oganov, A. R. Systematic search for low-enthalpy $\mathrm{sp}^{3}$ carbon allotropes using evolutionary metadynamics. Phys. Rev. B 85, 201407 (2012).

19. Zhu, Q., Oganov, A. R. \& Lyakhov, A. O. Evolutionary metadynamics: a novel method to predict crystal structures. CrystEngComm 14, 3596-3601 (2012).

20. Pickard, C. \& Needs, R. High-pressure phases of silane. Phys. Rev. Lett. 97, 045504 (2006).

21. Pickard, C. J. \& Needs, R. J. Highly compressed ammonia forms an ionic crystal. Nat. Mater. 7, 775-779 (2008).

22. Wang, Y., Lv, J., Zhu, L. \& Ma, Y. Crystal structure prediction via particle-swarm optimization. Phys. Rev. B 82, 094116 (2010).

23. Wang, Y., Lv, J., Zhu, L. \& Ma, Y. Calypso: A method for crystal structure prediction. Comput. Phys. Commun. 183, 2063-2070 (2012).

24. Dellago, C., Bolhuis, P. G., Csajka, F. S. \& Chandler, D. Transition path sampling and the calculation of rate constants. J. Chem. Phys. 108, 1964 (1998). 
25. Zhao, Z. et al. Novel superhard carbon: C-centered orthorhombic $\mathrm{C}_{8}$. Phys. Rev. Lett. 107, 215502 (2011).

26. Wang, Z. et al. A quenchable superhard carbon phase synthesized by cold compression of carbon nanotubes. Proc. Natl. Acad. Sci. U.S.A. 101, 13699-13702 (2004)

27. Li, Q. et al. Superhard monoclinic polymorph of carbon. Phys. Rev. Lett. 102, 175506 (2009).

28. Wang, Y., Panzik, J. E., Kiefer, B. \& Lee, K. K. M. Crystal structure of graphite under room-temperature compression and decompression. Sci. Rep. 2, 520 (2012).

29. Venkateswaran, U. et al. Probing the single-wall carbon nanotube bundle: Raman scattering under high pressure. Phys. Rev. B 59, 10928 (1999).

30. Teredesai, P. V. et al. Pressure-induced reversible transformation in single-wall carbon nanotube bundles studied by Raman spectroscopy. Chem. Phys. Lett. 319, 296-302 (2000).

31. Khabashesku, V. N. et al. Polymerization of single-wall carbon nanotubes under high pressures and high temperatures. J. Phys. Chem. B 106, 11155-11162 (2002).

32. Popov, M., Kyotani, M., Nemanich, R. \& Koga, Y. Superhard phase composed of single-wall carbon nanotubes. Phys. Rev. B 65, 033408 (2002).

33. Kawasaki, S. et al. XRD and TEM study of high pressure treated single-walled carbon nanotubes and $\mathrm{C}_{60}$-peapods. Carbon 43, 37-45 (2005).

34. Blank, V. et al. Nanostructured superhard carbon phase obtained under high pressure with shear deformation from single-wall nanotubes Hipco. Physica B: Condensed Matter 382, 58-64 (2006).

35. Kumar, R. S. et al. X-ray Raman scattering studies on $\mathrm{C}_{60}$ fullerenes and multiwalled carbon nanotubes under pressure. Diamond Relat. Mater. 16, 1250-1253 (2007).

36. Schultz, P. A., Leung, K. \& Stechel, E. B. Small rings and amorphous tetrahedral carbon. Phys. Rev. B 59, 733-741 (1999).

37. Yildirim, T., Gülseren, O., Kılıç, Ç. \& Ciraci, S. Pressure-induced interlinking of carbon nanotubes. Phys. Rev. B 62, 12648-12651 (2000)

38. Zhao, Z. et al. Three dimensional carbon-nanotube polymers. ACS Nano 5, 7226-7234 (2011).

39. Ouyang, M., Huang, J. L., Cheung, C. L. \& Lieber, C. M. Energy gaps in "metallic" single-walled carbon nanotubes. Science 292, 702-705 (2001).

40. Delaney, P., Choi, H. J., Ihm, J., Louie, S. G. \& Cohen, M. L. Broken symmetry and pseudogaps in ropes of carbon nanotubes. Nature 391, 466-468 (1998).

41. Hoffmann, R., Hughbanks, T., Kertesz, M. \& Bird, P. H. Hypothetical metallic allotrope of carbon. J. Am. Chem. Soc. 105, 4831-4832 (1983).

42. Tangney, P., Capaz, R. B., Spataru, C. D., Cohen, M. L. \& Louie, S. G. Structural transformations of carbon nanotubes under hydrostatic pressure. Nano Lett. 5, 2268-2273 (2005)

43. Sluiter, M. \& Kawazoe, Y. Phase diagram of single-wall carbon nanotube crystals under hydrostatic pressure. Phys. Rev. B 69, 224111 (2004).

44. Kılıç, Ç., Ciraci, S., Gülseren, O. \& Yildirim, T. Variable and reversible quantum structures on a single carbon nanotube. Phys. Rev. B 62, R16345-R16348 (2000).

45. Fahy, S., Louie, S. G. \& Cohen, M. L. Pseudopotential total-energy study of the transition from rhombohedral graphite to diamond. Phys. Rev. B 34, 1191 (1986).

46. Zhou, X.-F. et al. Ab initio study of the formation of transparent carbon under pressure. Phys. Rev. B 82, 134126 (2010).

47. Gao, F. et al. Hardness of covalent crystals. Phys. Rev. Lett. 91, 015502 (2003).

48. He, J., Wu, E., Wang, H., Liu, R. \& Tian, Y. Ionicities of boron-boron bonds in $\mathrm{B}_{12}$ icosahedra. Phys. Rev. Lett. 94, 015504 (2005).
49. Guo, X. et al. Hardness of covalent compounds: roles of metallic component and d valence electrons. J. Appl. Phys. 104, 023503 (2008).

50. Tian, Y., Xu, B. \& Zhao, Z. Microscopic theory of hardness and design of novel superhard crystals. Int. J. Refract. Met. Hard Mater. 33, 93-106 (2012).

51. Guo, X. et al. Unbinding force of chemical bonds and tensile strength in strong crystals. J. Phys.: Condens. Matter 21, 485405 (2009).

52. Demczyk, B. G. et al. Direct mechanical measurement of the tensile strength and elastic modulus of multiwalled carbon nanotubes. Mater. Sci. Eng. A 334, 173-178 (2002).

53. Frantsevich, I., Voronov, F. \& Bokuta, S. Elastic constants and elastic moduli of metals and insulators handbook. Naukova Dumka, Kiev, 60-180 (1983).

54. Pugh, S. F. Xcii. Relations between the elastic moduli and the plastic properties of polycrystalline pure metals. Phil. Mag. 45, 823-843 (1954)

55. Wang, J.-T., Chen, C. \& Kawazoe, Y. Low-temperature phase transformation from graphite to $\mathrm{sp}^{3}$ orthorhombic carbon. Phys. Rev. Lett. 106, 075501 (2011).

56. Clark, S. J. et al. First principles methods using CASTEP. Zeitschrift für Kristallographie 220, 567-570 (2005).

57. Ceperley, D. M. \& Alder, B. J. Ground state of the electron gas by a stochastic method. Phys. Rev. Lett. 45, 566-569 (1980).

58. Perdew, J. P. \& Zunger, A. Self-interaction correction to density-functional approximations for many-electron systems. Phys. Rev. B 23, 5048-5079 (1981).

59. Monkhorst, H. J. \& Pack, J. D. Special points for Brillouin-zone integrations. Phys. Rev. B 13, 5188-5192 (1976).

\section{Acknowledgments}

This work was supported by NBRPC (Grant No. 2011CB808205), NSFC (Grants No. 51121061, No. 91022029, and No. 51272227). A.R.O. acknowledges funding from the National Science Foundation (grant EAR-1114313), and access to computing facilities at the Center for Functional Nanomaterials (Brookhaven National Laboratory), Moscow State University (Russia), and at the Joint Supercomputer Center (Russian Academy of Sciences, Moscow, Russia).

\section{Author contributions}

B.X. and Y.J.T. designed and coordinated the overall study. M.H., Z.S.Z., F.T., Q.Q.W. and M.X. performed calculations. A.R.O., C.Z.F., B.W., J.L.H., D.L.Y., H.-T.W., B.X. and Y.J.T. analyzed all data. M.H., Z.S.Z., F.T., B.X. and Y.J.T. wrote the paper. All authors contributed to the discussion of the results.

\section{Additional information}

Supplementary information accompanies this paper at http://www.nature.com/ scientificreports

Competing financial interests: The authors declare no competing financial interests.

License: This work is licensed under a Creative Commons

Attribution-NonCommercial-NoDerivs 3.0 Unported License. To view a copy of this license, visit http://creativecommons.org/licenses/by-nc-nd/3.0/

How to cite this article: Hu, M. et al. Compressed carbon nanotubes: A family of new multifunctional carbon allotropes. Sci. Rep. 3, 1331; DOI:10.1038/srep01331 (2013). 